EGU2020-20907

https://doi.org/10.5194/egusphere-egu2020-20907

EGU General Assembly 2020

(c) Author(s) 2020. This work is distributed under

the Creative Commons Attribution 4.0 License.

\title{
Burned area trends in the Brazilian Cerrado: the roles of climate and anthropogenic drivers
}

\author{
Patrícia S. Silva ${ }^{1}$, Julia A. Rodrigues ${ }^{2}$, Filippe L. M. Santos ${ }^{2}$, Joana Nogueira ${ }^{2,3}$, Allan A. Pereira ${ }^{4}$, \\ Leonardo F. Peres ${ }^{2}$, Duarte Oom ${ }^{5}$, Carlos C. DaCamara ${ }^{1}$, José M. C. Pereira ${ }^{6}$, and Renata \\ Libonati ${ }^{1,2,6}$ \\ ${ }^{1}$ Instituto Dom Luiz (IDL), Faculdade de Ciências, Universidade de Lisboa, Campo Grande, 1749-016, Lisboa, Portugal \\ ${ }^{2}$ Departamento de Meteorologia, Universidade Federal do Rio de Janeiro, 21941-916, Rio de Janeiro, Brazil \\ ${ }^{3}$ Programa de Monitoramento de Queimada por Satélites, Instituto Nacional de Pesquisas Espaciais, São José dos Campos, \\ Brazil \\ ${ }^{4}$ Instituto Federal de Ciência e Tecnologia do Sul de Minas Gerais, 37890-000, Muzambinho, MG, Brazil \\ ${ }^{5}$ European Commission, Joint Research Centre (JRC), Ispra, Italy \\ ${ }^{6}$ Centro de Estudos Florestais (CEF), Instituto Superior de Agronomia, Universidade de Lisboa,1349-017, Lisboa, Portugal
}

Fire is a natural disturbance in the Brazilian savannas, Cerrado, with substantial ecological and economic impacts. Most studies have characterized the fire regime in this biome using climate drivers but neglected the geographical variation of anthropogenic activities. These factors can trigger inappropriate fire-fighting decisions and biodiversity conservation policies. This takes special relevance in fire-prone biomes with recent fire management policies as Cerrado, which have been highly modified over the last decades due to changes in land use and climate.

Here, we aim to identify how variations in climate and anthropogenic drivers influence burned area (BA) trends at the regional level (microregions) in Cerrado. We evaluated satellite-derived BA (MCD64, collection 6) for 172 microregions from 2001 to 2018 across the entire biome. The Canadian Forest Fire Weather Index (FWI) was used as a proxy of climate using meteorological variables from ECMWF's ERA5 reanalysis product. The human leverage, considered here as population density (PD) and land use (LU), were derived, respectively, from the annual census of the Brazillian Institute of Geography and Statistics (IBGE) and from a Brazilian platform of annual land use/cover mapping (MapBiomas). Recent BA trends considering the drivers FWI, LU and PD, were estimated using the non-parametric Theil-Sen regression and the modified Mann-Kendall test.

Results showed BA trends over the last 18 years were significant and spatially contrasted along Cerrado: positive trends were found in the north-eastern region (in particular, the most recent agricultural frontier in Brazil: MATOPIBA) whereas the south-western region showed negative trends. PD showed positive trends in all microregions and, similarly, LU obtained positive trends over most of Cerrado. Positive FWI trends were also found over the central and north-eastern regions and FWI was the driver that explained most of BA variance in Cerrado. LU and PD were found to have much more complex relations with BA. Moreover, regarding the seasonal variability 
of microregions with positive and negative trends, the former were found to begin earlier in June and last longer, indicating that the overall fire season in Cerrado may be extending.

The approach presented here allows the exploration of recent trends affecting fires, crucial to inform and support better allocation of resources in fire management under current and future conditions.

The study was funded by Conselho Nacional de Desenvolvimento Científico e Tecnológico, Brazil (CNPQ) through grants 305159/2018-6 and 441971/2018-0. P. Silva is funded by Fundação para a Ciência e a Tecnologia (FCT), grant number SFRH/BD/146646/2019. 